\title{
Cardy-like formula for the Schwarzschild black hole entropy
}

\author{
Mokhtar Hassaine* \\ Instituto de Matemática y Física, Universidad de Talca, Casilla 747, Talca, Chile
}

(Received 24 September 2019; accepted 29 March 2020; published 13 April 2020)

\begin{abstract}
The main part of this work is to present a formula allowing a derivation of the Schwarzschild black hole entropy in arbitrary dimension. More generally, this Cardy-like formula applies for static black holes whose gravitational entropy scales as a power $\alpha$ of the temperature, and is also effective for negative heat capacity solutions $\alpha<0$. The formula involves the scaling power $\alpha$, the black hole mass and the energy of a gravitational soliton. The robustness of this formula is verified in the most famous example of solution with negative heat capacity, namely the Schwarzschild black hole. The mass of the Schwarzschild regular soliton is computed using the counterterm method for asymptotically flat spacetimes. Corrections of the black hole entropy of the order of logarithm of the area are shown to arise for dimensions strictly greater than 4 .
\end{abstract}

DOI: 10.1103/PhysRevD.101.084028

\section{INTRODUCTION}

It is now accepted that black holes are thermodynamical objects endowed with a temperature and entropy [1,2]. A natural question has then emerged concerning the statistical interpretation of the black hole entropy. In the semiclassical approximation, the entropy is given by the famous Bekenstein-Hawking formula,

$$
\mathcal{S}_{\mathrm{BH}}=\frac{c^{3} k_{B} \mathcal{A}}{4 G \hbar},
$$

where $\mathcal{A}$ is the area of the event horizon of the black hole, $G$ is the Newton's constant, $\hbar$ the Planck's constant, $c$ the speed of light and $k_{B}$ the Boltzmann's constant. In what follows, for simplicity, we will set $c=k_{B}=\hbar=1$. The presence of the constants $G$ and $\hbar$ in this formula strongly suggests a connection between the quantum mechanics and gravity. Nevertheless, despite the fact that the area dependence of the black hole entropy (1) can be obtained from the quantum partition function for gravity [3], its relation to statistical mechanic is still rather mysterious. In fact, a deep understanding of the microscopic states responsible of the entropy area law will certainly provide important insights into quantum gravity. For this purpose, several interesting ideas have been put forward in the past decades to provide some possible explanations concerning the BekensteinHawking entropy. The first convincing counting of black

\footnotetext{
*hassaine-at-inst-mat.utalca.cl
}

Published by the American Physical Society under the terms of the Creative Commons Attribution 4.0 International license. Further distribution of this work must maintain attribution to the author(s) and the published article's title, journal citation, and DOI. Funded by SCOAP. hole microstates was given by Strominger and Vafa [4] in the case of extremal black holes in string theory. Later on, this computation was successfully extended to a wide variety of charged black holes in the extremal and nearextremal limit, see e.g., [5,6], as well as for nonextremal black holes through duality relations. There also exist many other microscopic derivations of black hole thermodynamics such as those exploiting the AdS/CFT correspondence [7] or those involving the loop quantum gravity [8], the entanglement entropy [9] or the asymptotic symmetries [10]; for an exhaustive list, see e.g., [11]. Despite that none of these proposals is completely satisfactory, these different approaches yield the same result, raising a problem of "universality" [12]. A certain universality is nevertheless effective in the case of two-dimensional conformal field theory where the entropy, in the high-energy regime, is fixed by symmetry independently of any details, and is given by the famous Cardy formula [13]:

$$
\mathcal{S} \sim \ln \rho(\Delta, \bar{\Delta}) \sim 2 \pi \sqrt{\frac{c_{\text {eff }} \Delta}{6}}+2 \pi \sqrt{\frac{\bar{c}_{\text {eff }} \bar{\Delta}}{6}} .
$$

Here, $c_{\text {eff }}=c-24 \Delta_{0}$ and $\bar{c}_{\text {eff }}=\bar{c}-24 \bar{\Delta}_{0}$ with $\Delta_{0}$ and $\bar{\Delta}_{0}$ being the lowest eigenvalues of the zero-mode Virasoro generators with central charges $c$ and $\bar{c}$. As proved by Brown and Henneaux [14], much before the advent of the AdS/CFT correspondence, the asymptotic symmetries of the three-dimensional AdS space consist in two copies of the Virasoro algebra with a central charge. Starting from this observation, it was shown by Strominger [10] that the Cardy formula (2) applied for the three-dimensional Banados-Teitelboim-Zanelli black hole [15] correctly reproduces the expression of the Bekenstein-Hawking entropy. This computation was also generalized in higher 
dimensions for black holes having a two-dimensional conformal field theory (CFT) dual in the case of standard general relativity [16], and also in the presence of higherderivative corrections [17].

The deepness of the Cardy formula is essentially due to the modular invariance which is a characteristic feature of two-dimensional CFTs only. In order to circumvent this dimensional restriction, a higher-dimensional extension of Eq. (2) was proposed by Verlinde [18]. This so-called Cardy-Verlinde formula, which relates the entropy of a CFT with a large central charge to its total energy and Casimir energy defined as the subextensive part of the energy, has been shown to hold for strongly coupled field theories having an AdS dual. From a holographic point of view, the Cardy-Verlinde formula was tested in various examples admitting AdS vacua with relative success [19]. For completeness, we also mention that a generalization of the Cardy-Verlinde formula for asymptotically flat black holes was proposed in Ref. [20].

In the past decade, the interest in extending holography to spacetimes that are not asymptotically AdS has considerably grown up. In this context, a Lifshitz version of the Cardy formula was proposed in [21] where the $S$-modular invariance or equivalently the low-high temperature duality arises as a direct consequence of the isomorphism between the two-dimensional Lifshitz algebras with dynamical exponents $z$ and $z^{-1}$. Using this duality, a precise formula for the asymptotic growth of the number of states was obtained in [21], and the robustness of this formula was tested in a wide variety of Lifshitz black holes [21-23].

More recently, great progress in the microscopic derivation of black hole entropy has been made possible thanks to the study of three-dimensional black holes by highlighting the prominent role played by the gravitational soliton in the generalizations of the Cardy formula [21-31]. This point of view is not in contradiction with the standard Cardy formula where the ground state is implicitly assumed to be the three-dimensional AdS spacetime. However, it is important to emphasize that this assumption is valid only in the vacuum sector and not in the hairy sector where the ground state may be different. In fact, as shown in Refs. [24-26], a naive application of the standard Cardy formula (2) for three-dimensional hairy black holes whose asymptotic symmetry group remains the conformal one, does not fit with the semiclassical hairy black hole entropy. This puzzle can be circumvented by first rewriting the Cardy formula (2) in terms of the vacuum energy rather than the central charge, and by identifying the vacuum energy with the mass of a gravitational soliton obtained from the black hole by means of a double Wick rotation as it occurs for the AdS soliton [32]. The importance played by the soliton to correctly reproduce the semiclassical entropy has also been put in light in the case of anisotropic black brane solutions [21-23,27,30], and for AdS black branes in arbitrary dimension [24-26,28,29,31]. This is precisely the road that we propose to explore in this paper by presenting a Cardy-like formula that perfectly reproduces the Bekenstein-Hawking formula (1) in the case of the Schwarzschild black hole solution. In order to achieve this task, we will explicitly construct the Schwarzschild gravitational soliton obtained through a double Wick rotation followed by a suitable rescaling in order to avoid conical singularity. In doing so, the resulting configuration will be shown to be smooth and devoid of any constant of integration fulfilling what the ground state is expected to be.

The plan of the paper is organized as follows. In the next section, we will derive a Cardy-like formula assuming that the partition function enjoys a certain modular symmetry, and that the ground state is given by a gravitational soliton. This formula involves the mass of the black hole as well as of the corresponding gravitational soliton. In Sec. III, we will present the $d$-dimensional gravitational soliton and compute its mass by means of the counterterm method. We will explicitly check that the Cardy-like formula reproduces the $d$-dimensional Schwarzschild entropy (1). Comments and concluding remarks will be reported in the last section. In particular we will compute the logarithmic corrections of the Schwarzschild black hole entropy.

\section{CARDY-LIKE FORMULA WITH SOLITON}

As already mentioned in the Introduction, generalizations of the Cardy formula where the ground state is identified with a gravitational soliton have been given and explicitly tested in the current literature [21-31]

For these previously mentioned cases, a precise formula for the density of states at fixed energy $\mathcal{M}$ was obtained under the assumption that the bulk gravitational soliton (identified with the ground state of the putative field theory) has a negative mass denoted by $\mathcal{M}_{\text {soliton }}<0$, ensuring in turn the existence of a gap in the spectrum. We have noticed that the different Cardy-like formulas reported in Refs. [21$31]$ in the case of static black holes ${ }^{1}$ can be generically written as

$$
\mathcal{S}=\frac{2 \pi(\alpha+1)}{\alpha^{\frac{\alpha}{\alpha+1}}}\left(-\mathcal{M}_{\text {soliton }}\right)^{\frac{1}{\alpha+1}} \mathcal{M}^{\frac{\alpha}{\alpha+1}},
$$

where the parameter $\alpha>0$ is nothing but the scaling dependence of the gravitational entropy $\mathcal{S}_{G}$ with respect to the temperature, i.e.,

$$
\mathcal{S}_{G} \sim T^{\alpha} .
$$

This is not surprising since it is known that the entropy of static anisotropic black branes or static AdS black branes satisfies this kind of law temperature dependence (4) with a

\footnotetext{
${ }^{1}$ For rotating AdS black branes, the formula is much more involved because of the presence of all the rotation generators, see $[28,29,31]$.
} 
positive scaling $\alpha>0$. This is a direct consequence of the fact that the horizon metric of these black holes is a Euclidean space with zero curvature. Note that for black holes satisfying (4), the parameter $\alpha$ is nothing but the quotient of the heat capacity by the entropy. Hence, the positivity of the parameter $\alpha$ for solutions satisfying (4) ensures as well the positivity of the heat capacity.

For later convenience, we review the derivation of the generic formula (3) following closely the steps described in Ref. [21]. At low temperature $T=\beta^{-1} \ll \epsilon$, the partition function $\mathcal{Z}[\beta]$ is dominated by the contribution of the soliton with negative mass $\mathcal{Z}[\beta] \approx e^{-\beta \mathcal{M}_{\text {soliton }}}$ while at high temperature it becomes

$$
\mathcal{Z}[\beta] \approx e^{-(2 \pi)^{1+\alpha} \beta^{-\alpha} \mathcal{M}_{\text {soliton }},}
$$

by assuming that the partition function is invariant under the following generalized modular transformation (with $\alpha>0$ ):

$$
\mathcal{Z}[\beta]=\mathcal{Z}\left[(2 \pi)^{1+\alpha} \beta^{-\alpha}\right] .
$$

For $\alpha=1$, this relation is nothing but the $S$-modular invariance for chiral movers in conformal field theory [13]. In general, for $\alpha>0$, the relation (5) reflects the low-high temperature duality as far as the ground state has a negative energy. Nevertheless, as shown below, this duality can also be effective for $\alpha<0$ but for a ground state with positive energy but still dominated by the mass of the black hole.

Using this duality relation, the asymptotic growth of number of states $\rho(\mathcal{M})$ at fixed mass $\mathcal{M}$ can be written as

$$
\begin{aligned}
\rho(\mathcal{M}) & \approx \int e^{f(\beta, \mathcal{M})} d \beta, \\
f(\beta, \mathcal{M}) & =\beta \mathcal{M}-(2 \pi)^{1+\alpha} \beta^{-\alpha} \mathcal{M}_{\text {soliton }},
\end{aligned}
$$

and can be evaluated in the saddle point approximation at the extremum of $f(\beta, \mathcal{M})$. In this case, the extremum is a minimum reached at the point

$$
\beta_{*}=2 \pi \alpha^{\frac{1}{\alpha+1}} \frac{\left(-\mathcal{M}_{\text {soliton }}\right)^{\frac{1}{\alpha+1}}}{\mathcal{M}^{\frac{1}{\alpha+1}}},
$$

and, hence the entropy $\mathcal{S}=\log \rho(\mathcal{M})$ at first approximation, $\mathcal{S} \approx f\left(\beta_{*}, \mathcal{M}\right)$, yields the formula (3).

It is clear from the expression (3) that the Cardy-like formula can be ill defined for certain negative values of the parameter $\alpha$. This is not surprising since as shown previously the derivation of (3), using the modular transformation (5), requires the parameter $\alpha$ to be strictly positive (unless the ground state has a positive energy). Nevertheless, there also exist physical interesting solutions with negative heat capacity. The most famous such example is given by the $d$-dimensional Schwarzschild black hole where the scaling parameter (4) is given by $\alpha=2-d<0$. Starting from this observation, we consider as important the necessity of extending the Cardy-like formula (3) for negative scaling parameter $\alpha$. We now see that a similar formula can be obtained for negative values of $\alpha$ assuming that the mass of the soliton is positive and such that $0<\mathcal{M}_{\text {soliton }}<\mathcal{M}$. In this situation, following the same steps as previously, we end up with the extremization of the same function (6b) which is now located at

$$
\beta_{*}=2 \pi(-\alpha)^{\frac{1}{\alpha+1}}\left(\frac{\mathcal{M}_{\text {soliton }}}{\mathcal{M}}\right)^{\frac{1}{\alpha+1}}
$$

As before, at the first approximation $\mathcal{S} \approx f\left(\beta_{*}, \mathcal{M}\right)$, one will get the following Cardy-like formula:

$$
\mathcal{S}=\frac{2 \pi(\alpha+1)}{\alpha}|\alpha|^{\frac{1}{\alpha+1}}\left(\mathcal{M}_{\text {soliton }}\right)^{\frac{1}{\alpha+1}} \mathcal{M}^{\frac{\alpha}{\alpha+1}} .
$$

There is however a notable difference with the positive $\alpha$ case. Indeed, for $\alpha<-1$ (as it occurs for the Schwarzschild solution), the extremum $\beta_{*}$ defined by Eq. (8) will correspond to a maximum, and consequently logarithmic corrections to the Bekenstein-Hawking entropy (1) will appear at the next order. More details concerning this point will be discussed in the end of the paper. For completeness, we mention that for the remaining negative values of $\alpha$, namely for $\alpha \in]-1,0[$, the extremum is a minimum, and the expression (9) will yield negative values of the entropy. We will also comment on this particular situation in the last section.

\section{SCHWARZSCHILD GRAVITATIONAL SOLITON}

The main objective of the present work is to check the validity of the Cardy-like formula (9) for black holes satisfying the condition (4) with negative values of $\alpha$. As recalled in the Introduction, the negativity of the scaling parameter $\alpha$ will correspond to black holes with negative heat capacity. The most famous known example is provided by the Schwarzschild black hole which will be our guiding example.

In arbitrary dimension $d \geq 4$, we consider the following regularized action:

$$
\begin{aligned}
I= & I_{\mathrm{bulk}}+I_{\mathrm{GH}}+I_{\mathrm{ct}} \\
= & \frac{1}{16 \pi G} \int_{M} d^{d} x \sqrt{-g} R+\frac{1}{8 \pi G} \int_{\partial M} d^{d-1} x \sqrt{-\gamma} K \\
& -\frac{(d-2)}{8 \pi G} \int_{\partial M} d^{d-1} x \sqrt{-\gamma} \sqrt{\frac{\tilde{R}}{(d-2)(d-3)}},
\end{aligned}
$$

where $K$ is the trace of the extrinsic curvature denoted by $K_{a b}, \gamma_{a b}$ is the induced metric on the boundary $\partial M$ with 
Ricci scalar curvature $\tilde{R}$. The regularized action is composed by the bulk action together with the standard Gibbons-Hawking boundary term $I_{\mathrm{GH}}$, and the last term is an appropriate counterterm for asymptotically flat spacetimes $[33,34]$. The Schwarzschild black hole solution with coordinates $\left(t, r, x_{1}, \ldots, x_{d-2}\right)$ is given by

$$
d s^{2}=-\left(1-\frac{r_{h}^{d-3}}{r^{d-3}}\right) d t^{2}+\frac{d r^{2}}{1-\frac{r_{h}^{d-3}}{r^{d-3}}}+r^{2} h_{i j}(x) d x^{i} d x^{j},
$$

where $r_{h}$ is the location of the horizon, and the unit $(d-2)$ dimensional spherical metric is parametrized as

$$
h_{i j}(x) d x^{i} d x^{j}=\sum_{k=1}^{d-3}\left(\prod_{j=k+1}^{d-2} \sin ^{2}\left(x_{j}\right)\right) d x_{k}^{2}+d x_{d-2}^{2} .
$$

The Schwarzschild mass $\mathcal{M}$ and gravitational entropy $\mathcal{S}_{G}$ can be computed from the regularized action (10) yielding the well-known results,

$$
\begin{aligned}
& \mathcal{M}=\frac{(d-2) r_{h}^{d-3}}{16 \pi G}\left|\Omega_{d-2}\right|, \\
& \mathcal{S}_{G}=\frac{r_{h}^{d-2}}{4 G}\left|\Omega_{d-2}\right|,
\end{aligned}
$$

with $\left|\Omega_{d-2}\right|=\int d^{d-2} x \sqrt{h}$. On the other hand, the temperature is inversely proportional to the horizon location

$$
T=\frac{(d-3)}{4 \pi r_{h}},
$$

and consequently the scaling parameter $\alpha$ as defined by Eq. (4) is negative and given by $\alpha=2-d$.

In order to check the validity of the Cardy-like formula (9) with this scaling parameter, we need to derive the gravitational soliton and compute its mass. The regular soliton obtained from the Schwarzschild solution by a double Wick rotation given by $t \rightarrow i x_{1}$ and $x_{1} \rightarrow i t$ followed by a suitable rescaling that permits to absorb the constant of integration reads

$$
\begin{aligned}
d s^{2}= & -\frac{(d-3)^{2}}{4} \cosh ^{\frac{4}{d-3}}(\rho) \prod_{j=2}^{d-2} \sin ^{2}\left(x_{j}\right) d t^{2} \\
& +\cosh ^{\frac{4}{d-3}}(\rho) d \rho^{2}+\tanh ^{2}(\rho) d x_{1}^{2}+\frac{(d-3)^{2}}{4} \cosh ^{\frac{4}{d-3}}(\rho) \\
& \times\left[\sum_{k=2}^{d-3}\left(\prod_{j=k+1}^{d-2} \sin ^{2}\left(x_{j}\right)\right) d x_{k}^{2}+d x_{d-2}^{2}\right]
\end{aligned}
$$

One can appreciate the regularity of this spacetime (13) by noting that its Kretchmann invariant,

$$
K=R_{\alpha \beta \sigma \gamma} R^{\alpha \beta \sigma \gamma}=\frac{16(d-1)(d-2)^{2}}{(d-3)^{3} \cosh ^{\frac{4(d-1)}{(d-3)}}(\rho)},
$$

is regular everywhere for $d>3$. After some tedious but straightforward computations, the mass of the soliton $\mathcal{M}_{\text {soliton }}$ is computed from the regularized action (10), once evaluated in the Euclidean continuation of the soliton (13), yielding

$$
\mathcal{M}_{\text {soliton }}=\frac{1}{8 \pi G} \frac{(d-3)^{d-3}}{2^{d-2}}\left|\Omega_{d-2}\right|
$$

As anticipated, we notice that the soliton mass turns out to be always positive. Now, it is easy to corroborate that the Cardy-like formula (9) with a scaling parameter $\alpha=2-d$, and with the black hole and soliton masses [(11a)-(14)] correctly reproduces the Schwarzschild black hole entropy (11b) or equivalently the Bekenstein-Hawking formula (1).

\section{COMMENTS AND CONCLUDING REMARKS}

The two formulas (3) and (9) which apply respectively for $\alpha>0$ with a negative mass soliton $\mathcal{M}_{\text {soliton }}<0$, and for $\alpha<0$ with $\mathcal{M}_{\text {soliton }}>0$, can be combined in a single formula that holds for any nonzero $\alpha$ as

$$
\mathcal{S}=\frac{2 \pi(\alpha+1)}{\alpha}|\alpha|^{\frac{1}{\alpha+1}}\left|\mathcal{M}_{\text {soliton }}\right|^{\frac{1}{\alpha+1}} \mathcal{M}^{\frac{\alpha}{\alpha+1}} .
$$

The robustness of this generic Cardy-like formula (15) for static black holes satisfying the scaling condition $\mathcal{S} \propto T^{\alpha}$ has been verified:

(i) previously, for black branes with different asymptotics (AdS, Lifshitz, hyperscaling violation), and with positive values of the parameter $\alpha$ in Refs. [21-31],

(ii) and in the present work for negative values of $\alpha$ in the case of the asymptotically flat Schwarzschild black hole solution.

In all these working examples, the gravitational entropy is proportional to area of the horizon $\mathcal{S}_{G} \propto \mathcal{A}$, and hence this last condition can be thought to be necessary for the validity of the formula (15). It will be interesting to clarify this point by checking the validness or not of the formula (15) for black holes whose entropy satisfies our working hypothesis (4), but without being proportional to the area of the horizon. One such example is provided by a family of asymptotically flat Lovelock black holes [35] parametrized by an integer $k=\left\{1,2, \ldots,\left[\frac{d-1}{2}\right]\right\}$ whose metrics,

$$
\begin{aligned}
d s^{2}= & -\left(1-\frac{r_{h}^{d-2 k-1}}{r^{d-2 k-1}}\right) d t^{2}+\frac{d r^{2}}{1-\left(\frac{r_{h}}{r}\right)^{d-2 k-1}} \\
& +r^{2}\left[\sum_{p=1}^{d-3}\left(\prod_{j=p+1}^{d-2} \sin ^{2}\left(x_{j}\right)\right) d x_{p}^{2}+d x_{d-2}^{2}\right]
\end{aligned}
$$


are solutions to the field equations associated to the $d$ dimensional actions labeled by the integer $k$, and defined by

$$
I_{k}=\int_{M} \epsilon_{a_{1} \cdots a_{d}} R^{a_{1} a_{2}} \cdots R^{a_{2 k-1} a_{2 k}} e^{a_{2 k+1}} \cdots e^{a_{d}} .
$$

Here $e^{a}$ is the vielbein, $R^{a b}=d \omega^{a b}+\omega_{c}^{a} \omega^{c b}$ stands for the curvature two-form, and the wedge products between the forms are voluntarily omitted for simplicity. For these asymptotically flat Lovelock solutions, the gravitational entropy is given by $\mathcal{S} \propto r_{h}^{d-2 k}$, and hence it will be proportional to the area of the horizon only in the Einstein-Hilbert case $k=1$. In a forthcoming work, we will precisely study the microscopic derivation of Lovelock black hole entropy in the asymptotically flat case as well as in the presence of a cosmological constant [36]. The interest of such work is also motivated by the fact that the Cardy-Verlinde formula [18] has been shown to fail for the AdS Lovelock black holes [19], even in the large horizon radius limit where the AdS Lovelock entropy becomes proportional to the area of the horizon. Note that in this regime, i.e., $r_{h} \gg \epsilon$, the AdS Lovelock entropy satisfies as well our working hypothesis (4), and consequently it will be interesting to check the validity of the formula reported here (15).

Another point to be clarified is that for $\alpha<0$ in the range $\alpha \in]-1,0$ [, the expressions (9) or (15) will yield negative values of the entropy. One could hence think that the range of the possible values of $\alpha$ must exclude the interval ] $-1,0[$, but fortunately there exist black hole solutions with negative entropy in the current literature, see e.g., [3739]. One such example is given by a solution of the fivedimensional vacuum Einstein equations, $R_{\mu \nu}=0$, with a horizon topology modeled by the so-called Nil geometry (which is one of the eight geometries of the three-dimensional Thurston classification [40]). More precisely, the Nil black hole metric, solution of the vacuum Einstein equations, is given by [37]

$$
\begin{aligned}
d s^{2}= & -\left(1-r_{h} / r\right) d t^{2}+\frac{d r^{2}}{r^{5}\left(1-r_{h} / r\right)}+\frac{1}{r}\left(d x_{1}^{2}+d x_{2}^{2}\right) \\
& +r\left(d x_{3}-x_{1} d x_{2}\right)^{2},
\end{aligned}
$$

and its mass, entropy and temperature computed by the Euclidean method yield [37]

$$
\mathcal{M}=\frac{\left|\Omega_{3}\right|}{32 \pi G} r_{h}, \quad \mathcal{S}=-\frac{\left|\Omega_{3}\right|}{4 G \sqrt{r_{h}}}, \quad T=\frac{r_{h}^{\frac{3}{2}}}{4 \pi} .
$$

It is clear from these expressions that the scaling parameter as defined by Eq. (4) is given by $\alpha=-\frac{1}{3}$, and hence from the multiplying factor of the entropy formula (9), namely $(\alpha+1) / \alpha$, this would yield to a negative entropy in complete accordance with (17). In addition, one can note that the mass term in the Cardy-like formula (9), i.e., $\mathcal{M}^{\alpha /(\alpha+1)}$, will reproduce the correct horizon dependence of the entropy $\mathcal{S} \propto r_{h}^{-1 / 2}$. Now, in order to fully corroborate the formula (9), it will remain to identify the ground state and to compute its mass. However, it is evident from the Nil black hole solution (16) that any Wick rotation will drastically change the signature of the solution. Consequently, the recognition of the appropriate ground state which seems to be far from obvious should deserve some attention.

A nontrivial task will be to extend this derivation for the Kerr solution in four dimensions and its extension in higher dimension $d$ involving all the different rotation generators $\mathcal{J}_{i}$, with $1 \leq i \leq[(d-1) / 2]$.

Finally, another interesting problem is concerned by the subleading corrections of the black hole entropy (1) which are known to be proportional to the logarithm of the area [41]. For negative values of $\alpha$, we have seen that $\beta_{*}$ defined by Eq. (8) corresponds to a maximum of the function $f$ defined in (6b). Consequently, a Taylor expansion at second order of the integrand of the asymptotic growth of number of states (6a) will yield

$$
\mathcal{S} \approx f\left(\beta_{*}, \mathcal{M}\right)-\frac{1}{2} \log \left[\frac{\alpha(\alpha+1) \mathcal{M}^{\frac{\alpha+2}{\alpha+1}}}{\left(\mathcal{M}_{\text {soliton }}\right)^{\frac{1}{\alpha+1}}}\right],
$$

and, in the Schwarzschild case, this expression becomes

$$
\mathcal{S} \approx \frac{\mathcal{A}}{4 G}-\frac{1}{2}\left(\frac{d-4}{d-2}\right) \log (\mathcal{A})-\frac{1}{2} \log (\gamma)
$$

where $\gamma$ is an area-independent factor given by

$$
\gamma=\frac{(d-2)(d-3)^{2}}{32 \pi G}\left|\Omega_{d-2}\right|^{\frac{2}{d-2}} .
$$

It is interesting to note that the corrections of order of the logarithm of the area only appear for dimensions strictly greater than 4 . Indeed, for the four-dimensional Schwarzschild metric, the entropy approximation (18) reduces to

$$
\mathcal{S} \approx \frac{\mathcal{A}}{4 G}-\frac{1}{2} \log \left(\frac{1}{4 G}\right)
$$

\section{ACKNOWLEDGMENTS}

This work is partially supported by a grant from ECOSCONICYT 180011. 
[1] J. D. Bekenstein, Phys. Rev. D 7, 2333 (1973).

[2] S. W. Hawking, Commun. Math. Phys. 43, 199 (1975); 46, 206(E) (1976).

[3] G. W. Gibbons and S. W. Hawking, Phys. Rev. D 15, 2752 (1977).

[4] A. Strominger and C. Vafa, Phys. Lett. B 379, 99 (1996).

[5] C. G. Callan and J. M. Maldacena, Nucl. Phys. B472, 591 (1996).

[6] G. T. Horowitz and A. Strominger, Phys. Rev. Lett. 77, 2368 (1996).

[7] O. Aharony, S. S. Gubser, J. M. Maldacena, H. Ooguri, and Y. Oz, Phys. Rep. 323, 183 (2000).

[8] M. Domagala and J. Lewandowski, Classical Quantum Gravity 21, 5233 (2004).

[9] L. Bombelli, R. K. Koul, J. Lee, and R. D. Sorkin, Phys. Rev. D 34, 373 (1986).

[10] A. Strominger, J. High Energy Phys. 02 (1998) 009.

[11] S. Carlip, Lect. Notes Phys. 769, 89 (2009).

[12] S. Carlip, J. Phys. Conf. Ser. 67, 012022 (2007).

[13] J. L. Cardy, Nucl. Phys. B270, 186 (1986).

[14] J. D. Brown and M. Henneaux, Commun. Math. Phys. 104, 207 (1986).

[15] M. Banados, C. Teitelboim, and J. Zanelli, Phys. Rev. Lett. 69, 1849 (1992).

[16] M. Guica, T. Hartman, W. Song, and A. Strominger, Phys. Rev. D 80, 124008 (2009).

[17] T. Azeyanagi, G. Compere, N. Ogawa, Y. Tachikawa, and S. Terashima, Prog. Theor. Phys. 122, 355 (2009).

[18] E. P. Verlinde, arXiv:hep-th/0008140.

[19] R. G. Cai, Phys. Rev. D 63, 124018 (2001).

[20] D. Klemm, A. C. Petkou, G. Siopsis, and D. Zanon, Nucl. Phys. B620, 519 (2002).

[21] H. A. Gonzalez, D. Tempo, and R. Troncoso, J. High Energy Phys. 11 (2011) 066.

[22] E. Ayon-Beato, M. Bravo-Gaete, F. Correa, M. Hassaine, M. M. Juarez-Aubry, and J. Oliva, Phys. Rev. D 91, 064006 (2015).
[23] E. Ayon-Beato, M. Bravo-Gaete, F. Correa, M. Hassaine, and M. M. Juarez-Aubry, Phys. Rev. D 100, 044024 (2019).

[24] F. Correa, C. Martinez, and R. Troncoso, J. High Energy Phys. 01 (2011) 034.

[25] F. Correa, C. Martinez, and R. Troncoso, J. High Energy Phys. 02 (2012) 136.

[26] F. Correa, A. Faundez, and C. Martinez, Phys. Rev. D 87, 027502 (2013).

[27] E. Shaghoulian, J. High Energy Phys. 11 (2015) 081.

[28] E. Shaghoulian, Phys. Rev. D 93, 126005 (2016).

[29] E. Shaghoulian, Phys. Rev. D 94, 104044 (2016).

[30] M. Bravo-Gaete, S. Gomez, and M. Hassaine, Phys. Rev. D 91, 124038 (2015); 92, 124002 (2015).

[31] M. Bravo-Gaete, L. Guajardo, and M. Hassaine, J. High Energy Phys. 04 (2017) 092.

[32] G. T. Horowitz and R. C. Myers, Phys. Rev. D 59, 026005 (1998).

[33] R. B. Mann, Phys. Rev. D 60, 104047 (1999).

[34] P. Kraus, F. Larsen, and R. Siebelink, Nucl. Phys. B563, 259 (1999).

[35] J. Crisostomo, R. Troncoso, and J. Zanelli, Phys. Rev. D 62 , 084013 (2000).

[36] M. Hassaine (to be published).

[37] M. Hassaine, Phys. Rev. D 91, 084054 (2015).

[38] M. Chernicoff, M. Galante, G. Giribet, A. Goya, M. Leoni, J. Oliva, and G. Perez-Nadal, J. High Energy Phys. 06 (2016) 159.

[39] A. Cisterna, L. Guajardo, and M. Hassaine, Eur. Phys. J. C 79, 418 (2019); 79, 710(E) (2019).

[40] W. P. Thurston Three-Dimensional Geometry and Topology, edited by S. Levy (Princeton University Press, Princeton, NJ, 1997).

[41] R. K. Kaul and P. Majumdar, Phys. Rev. Lett. 84, 5255 (2000). 